Title:

\title{
Automatic Cough Segmentation from Non-Contact Sound Recordings in Pediatric Wards
}

\section{Authors:}

Yusuf A Amrulloh*

School of Information Technology and Electrical Engineering, the University of Queensland, Brisbane, Australia.

Udantha R Abeyratne

School of Information Technology and Electrical Engineering, the University of Queensland, Brisbane, Australia.

Vinayak Swarnkar

School of Information Technology and Electrical Engineering, the University of Queensland, Brisbane, Australia.

\section{Rina Triasih}

Department of Child Health, Sardjito Hospital, Universitas Gadjah Mada, Yogyakarta, Indonesia.

Amalia Setyati

Department of Child Health, Sardjito Hospital, Universitas Gadjah Mada, Yogyakarta, Indonesia.

\section{Correspondence:}

Udantha R Abeyratne

78 School of Information Technology and Electrical Engineering, GP South, Staff House Road, The University of Queensland, Brisbane, Australia.

Phone: +61 7 33469063, Fax: +61 7 33654999, Email: udantha@itee.uq.edu.au

\section{Conflict of Interest declaration:}

We wish to confirm that there are no known conflicts of interest associated with this Publication.

* At present author is with Department of Electrical Engineering, Faculty of Industrial Engineering, Universitas Islam Indonesia, Yogyakarta, Indonesia 


\begin{abstract}
Cough is a common symptom of almost all childhood respiratory diseases. In a typical consultation session, physicians may seek for qualitative information (e.g. wetness) and quantitative information (e.g. cough frequency) either by listening to voluntary coughs or by interviewing the patients/carers. This information is useful in the differential diagnosis and in assessing the treatment outcome of the disease. The manual cough assessment is tedious, subjective, and not suitable for long-term recording. Researchers have attempted to develop automated systems for cough assessment but none of the existing systems have specifically targeted the pediatric population. In this paper we address these issues and develop a method to automatically identify cough segments from the pediatric sound recordings. Our method is based on extracting mathematical features such as non-Gaussianity, Shannon entropy, and cepstral coefficients to describe cough characteristics. These features were then used to train an Artificial Neural Network to detect coughs segment in the sound recordings. Working on a prospective data set of 14 subjects (sound recording length 840 minutes), proposed method achieved sensitivity, specificity, and Cohen's Kappa of 93\%, as an automated pediatric cough counting device as well as the front-end of a cough analysis system.
\end{abstract}

Keywords: pediatric respiratory diseases, cough, automatic cough segmentation,

\title{
I. INTRODUCTION
}

$98 \%$, and 0.65 , respectively. These results indicate that the proposed method has the potential to be developed

Cough is a defense mechanism of the body to clear the respiratory tract from foreign materials which are inhaled accidentally or produced internally by infections [1]. It is a common symptom appearing in early to mid stages of respiratory diseases such as pneumonia, the leading cause of death in children less than five years of age. It has been estimated that 
pneumonia causes over 1.6 million deaths in this group per year [2], with more than $97 \%$ of cases occurring in the developing countries [3]. The world health organization (WHO) also reported that in those countries, pertussis (whooping cough) has also become one of the major childhood morbidities with an estimated 50 million cases and 300,000 deaths every year [4].

Even though cough is common in respiratory diseases and considered as an important clinical symptom, there is no gold standard to assess it. In a typical consultation session, physicians may listen to several episodes of spontaneous or voluntary coughs, to obtain qualitative information such as the "wetness" of a cough. Such qualitative information is extremely useful in diagnosis as well as the treatment of respiratory diseases.

During consultation sessions physicians may also seek quantitative information on coughs, such as the frequency of occurrence of cough events over a given time interval. This information can be used to determine the nature (e.g., acute, chronic) and the severity of coughs as well as to monitor the efficacy of a treatment [5]. However, to obtain this information, physicians heavily rely on subjective reports of patients or their carers. There is a great need for an automated device capable of counting the number of coughs, especially in childhood diseases. More importantly, technology capable of automatically extracting cough events from pediatric recordings is urgently needed in order to facilitate the diagnosis of diseases such as pneumonia $[6,7]$.

Several approaches have been taken to develop automated cough counting systems (e.g., Hull Automatic Cough Counter (HACC), Leicester Cough Monitor (LCM), LifeShirt, VitaloJAK, and PulmoTrack). The performances of these devices are varied. The HACC claimed 80 percent sensitivity and 96 percent specificity [8]. The figures for LifeShirt, Pulmotrack, LCM, and Vitalojak are (78\%, 99\%), (94\%, 96\%), (85.7\%, 99.9\%), and (97.5\%, 97.7\%) respectively [9-12]. To extract the sound events, HACC computed the standard deviation of cough sound intensities [8], while VitaloJAK [13] and LCM [11, 14] used sound 
intensities themselves. This makes these methods susceptible to variations in recording conditions, such as the distance from the microphone to the patient, sensitivity of the recording instruments and the sound level of the coughs being recorded. Both LifeShirt [15] and PulmoTrack [16] described cough as having a distinguishable humped structure in the waveform. Then they identified coughs by counting the number and/or measuring the slope of these humps. None of these commercial devices have been tested on pediatric populations or subjects with diseases such as pneumonia. In such subjects, the cough sound intensities and waveform-shapes may vary widely. Therefore intensity or simple waveform-shape based methods are unlikely to be optimal and are less likely to be robust in field use.

Cough recording on children, especially the younger ones, pose several additional challenges. Younger children are unable to produce voluntary coughs upon request. Any method targeting pediatric populations should be capable of using spontaneous coughs recorded over a period of interest. In pediatric recordings, crying, vocalization, and grunting are found abundantly, intermixed with cough sounds. Consequently, technology developed for adults are unlikely to be optimal for use on children.

Existing commercial cough counting devices such as LifeShirt, Vitalojak, and Pulmotrack employ contact sensors. While the use of contact sensors may have some advantages, they also carry several drawbacks. Contact sensors, compared to non-contact (free-air) microphones are robust against background sound propagated through air. However, they are more vulnerable to sound conducted through tissue and bones; spurious rubbing sounds due to sensor movement can also be an issue. In infectious diseases, elaborate efforts are needed to avoid cross contamination of patients through contact instrumentation. Furthermore, in pediatric subjects, contact sensors can also be difficult to attach because of patient discomfort. In this paper we address these issues and propose a novel technology for the automated segmentation of cough 
events from recordings obtained using non-contact microphones in a pediatric ward. In particular:

- We design our algorithms to target the pediatric population (age $<6$ years), addressing a fundamental gap in current technology.

- We develop a method for the segmentation of cough events, with algorithms capable of discounting background sounds such as crying, vocalization, and grunting.

- We develop new techniques that are robust against the variation of cough sound intensity levels and waveform-shapes. This is an unprecedented approach in this field, to the best of our knowledge.

The method has the potential to be developed as an automated cough counting device as well as the front-end of a cough based diagnostic system.

\section{MATERIAL AND METHOD}

\subsection{Cough recording protocol}

The cough recording system consisted of a low-noise microphone having a cardioid beam pattern (Model NT3, RODE ${ }^{\circledR}$, Sydney, Australia), followed by a pre-amplifier and an A/D converter (Model Mobile Pre-USB, M-Audio ${ }^{\circledR}$, CA, USA). The output of the Mobile Pre-USB was connected to the USB port of a laptop computer. The nominal distance from the microphones to the mouth of subjects was $50 \mathrm{~cm}$. The actual distance could vary from $40 \mathrm{~cm}$ to $100 \mathrm{~cm}$ due to the subject movement. We kept the sampling rate at $F_{s}=44.1 \mathrm{k} \mathrm{samples} / \mathrm{s}$ and 16-bit resolution to obtain the best sound quality.

The data for this work were recorded at Sardjito hospital, Yogyakarta, Indonesia, from pediatric patients admitted on respiratory complaints. The inclusion criteria used in the recruitment was patients with at least two of the following symptoms: cough, sputum, breathlessness, and temperature higher than $37.5^{\circ} \mathrm{C}$. We excluded patients having advanced 
disease where recovery is not expected, diseases with droplet precautions and patients undergoing ventilation treatment. The recordings were started after physicians had examined the subjects, begun the initial treatment, and informed consent had been completed. We acquired data in the natural hospital environment, without modifying it anyway, other than placing our sound recording system by the bed (see Fig 1). The duration of recording for each subject was between four and six hours. The research protocol had received ethics clearances from Sardjito Hospital and The University of Queensland, Australia.

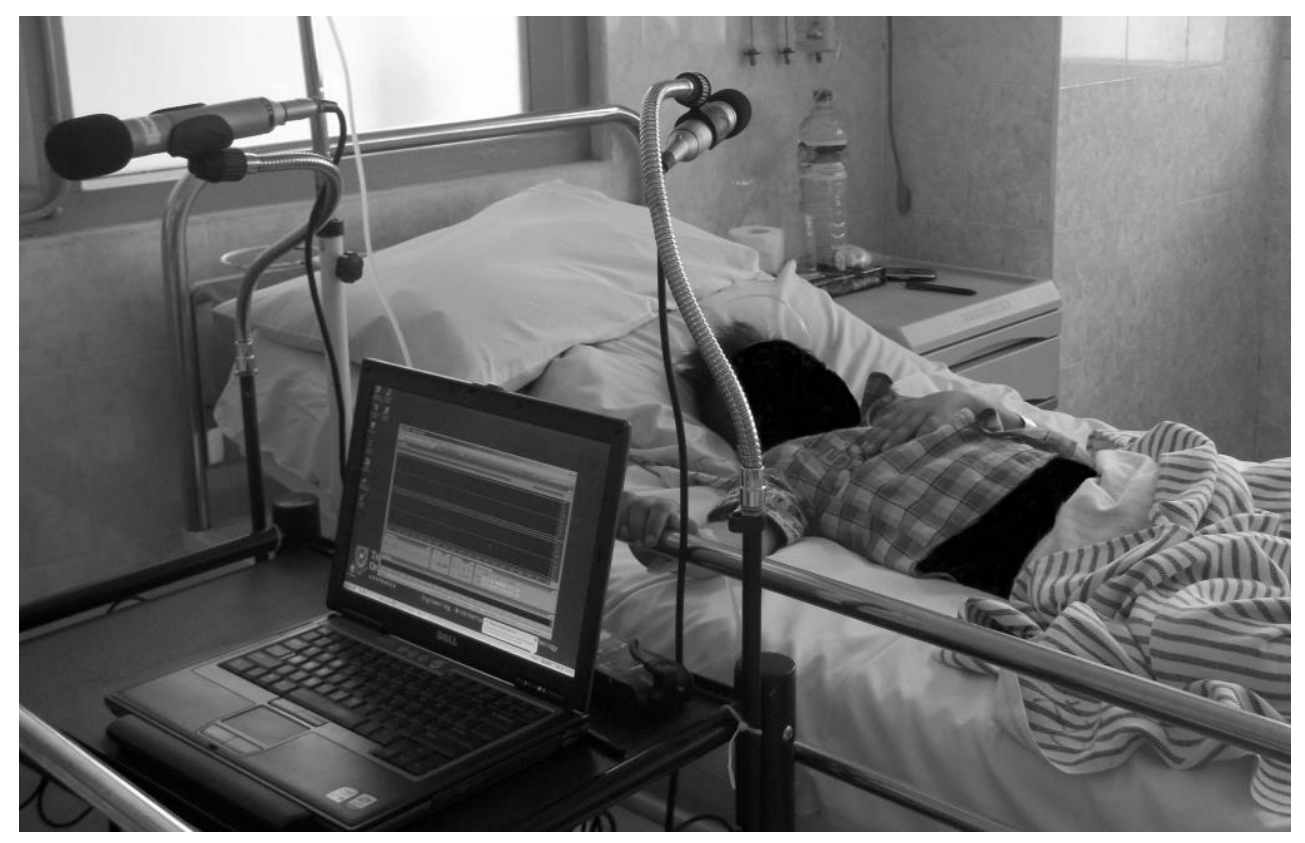

Figure 1. Cough recording system set up.

\subsection{Database and classification of cough segments}

In this paper, we used recordings from 24 pediatric subjects. We divided these subjects into two datasets namely:

(i). Model Design Dataset (MDD): Dataset MDD denotes the training dataset that was used to train the neural network $(\mathrm{NN})$ classifier. The main criterion in designing MDD is that it should contain the whole range of cough types and their variations as well as non-cough sounds expected in the practical setting. That way, the NN can learn the characteristics of 
the variety of coughs and learn to differentiate them from non-cough sounds. Data set MDD was designed by manually picking representative cough and non-cough sounds from each subject in the training dataset. All such sound events were then concatenated as a single data stream to form the set MDD. Thus, the dataset MDD is not a natural sequence of sound events, but the combination of a large number of handpicked cough and non-cough sounds. To develop MDD, we took cough and non-cough sound samples from $D_{l}=10$ subjects. The overall length of MDD was 15 minutes.

(ii). Prospective study dataset (PSD): Dataset PSD denotes the testing dataset and contains the actual sound stream recorded in the hospital. This way, our results on PSD can be taken as a true indication of performance in the clinical environment. Testing set PSD included the first 60 minutes of the sound streams recorded from $D_{2}=14$ subjects in the testing set. The total duration of PSD was 840 minutes.

Subjects in dataset MDD and PSD were mutually exclusive. The division of the subjects in two datasets was based on the order of presentation to the respiratory clinic of the hospital.

In both MDD and PSD, cough events were manually identified and marked. To define the beginning and end of cough segments, the scorer carefully listened to the sounds and simultaneously looked at the time domain waveform displayed on computer screen. Adobe Audition version 2.0 software was used in this process. This manual identification of cough events was used as the gold standard against which results of automatic classification were compared.

\subsection{A framework for cough sound analysis}

Based on physiological considerations, cough sounds are often considered as consisting of four different phases [1]: inspiratory, contractive, compressive, and expulsive. The inspiratory phase is initiated by breathing in and is terminated by the closure of the glottis. In the contractive phase, groups of respiratory muscles contract leading to a marked elevation of 
alveolar, pleural, and subglottic airway pressures. In the expulsive phase, the glottis opens quickly followed by rapid exhalation of air under a large pressure gradient. The rapid movement of air expelled from the lung generates the cough sounds with contributions coming from different areas of the respiratory system. The mechanism of cough sound production shares some similarities to that of speech production.

In this paper, inspired by human speech and snore processing techniques, we developed techniques for the analysis of cough sounds.

\subsection{Cough segmentation method}

A discretized sound recording $s[n]$ in MDD and PSD, can be modeled by the following equation:

$$
s[n]=s_{c g}[n]+s_{n c}[n]+b_{1}[n]+b_{2}[n]
$$

where $s_{c g}[n]$ is the cough sounds and $s_{n c}[n]$ is the non-cough sounds such as speech, cry, laughs, vocalization and sounds from hospital equipment. The quantity $b_{1}[n]$ represents the low frequency noise in the measurement (e.g.: noise coming from the vibration of microphone's stands) while $b_{2}[n]$ is the Gaussian noise (mostly high frequency).

In this paper, we propose an automated method to extract cough sounds $s_{c g}[n]$ from the recording $s[n]$. The block diagram of the overall method is shown in Fig 2. It comprises four main processes: noise reduction, feature extraction from sub-blocks of data, design of automatic classification model for classifying the sub-blocks into cough (CG) and non-cough (NC) classes, and identification of cough events by grouping CG/NC-classified contiguous subblocks of data.

In Sections 2.4.1 - 2.4.4, we provide a detailed description of the method.

\subsubsection{Noise reduction}


To reduce the noise $b_{1}[n]$ and $b_{2}[n]$, we processed $s[n]$ through two different filters: (a) a high pass filter (HPF), and, (b) a power spectral subtractions (PSS) filter. The HPF was implemented to reduce the low frequency noise $b_{1}[n]$. We designed the HPF as a fourth-order
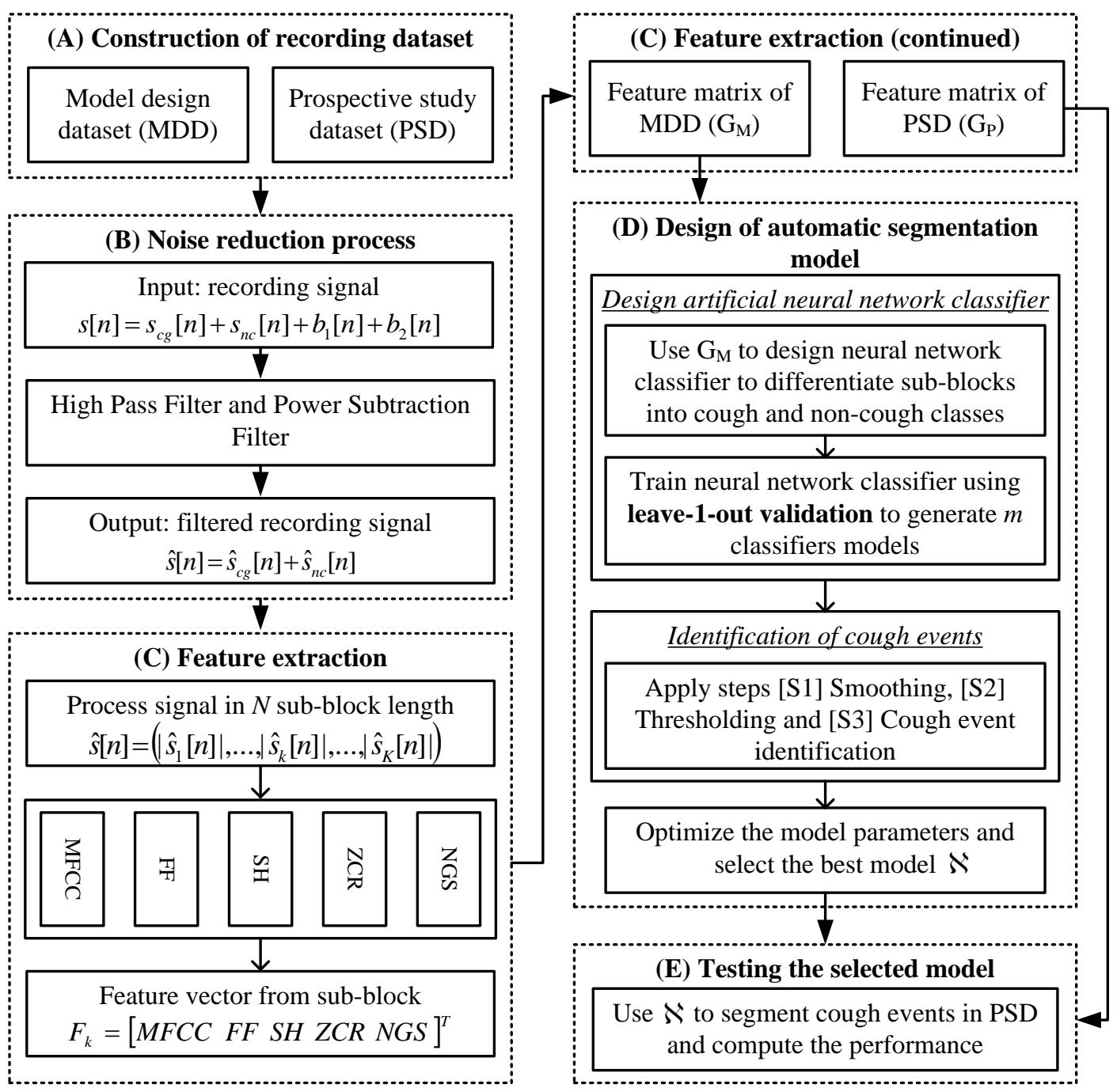

Figure 2. Block diagram of the proposed algorithm. It is comprised of five processes: (A) Construction of recording dataset, (B) Noise reduction, (C) Feature extraction, (D) Design of automatic classification model and (E) Testing the selected model in PSD.

Butterworth filter with cut off frequency $f_{c}=10 \mathrm{~Hz}$. The particular $f_{c}$ was selected based on the low frequency noise profile in dataset MDD. The PSS filter was employed to reduce the Gaussian noise $b_{2}[n]$ [17]. After the HPF and PSS filters, the estimate $\hat{s}[n]$ of the recording $s[n]$ is given by:

$$
\hat{s}[n]=\hat{s}_{c g}[n]+\hat{s}_{n c}[n]
$$


where $\hat{s}_{c g}[n]$ and $\hat{s}_{n c}[n]$ represent the estimates of cough sounds and non-cough sounds respectively.

Our signal of interest is the cough sound estimate $\hat{s}_{c g}[n]$. In the following we discuss the method used to extract $\hat{s}_{c g}[n]$ from the estimate $\hat{s}[n]$. The approach we take here is to extract from $\hat{s}[n]$ feature characteristic of $\hat{s}_{c g}[n]$ and use them to differentiate $\hat{s}_{c g}[n]$ and $\hat{s}_{n c}[n]$.

\subsubsection{Feature extraction of sound signal from the filtered signal $\hat{s}[n]$}

To obtain the features of the sound signal, we applied a rectangular sliding window $w_{r}[n]$ of length $N(N=882$ samples, equal to $20 \mathrm{~ms})$ to $\hat{s}[n]$, generating data sub-blocks. Let $\hat{s}[n]=\{\mid$ $\left.\hat{s}_{1}[n]|, \ldots,| \hat{s}_{k}[n]|, \ldots,| \hat{s}_{K}[n] \mid\right\}$ represents the filtered sound recording where $\hat{s}_{k}[n]$ represents the $k^{\text {th }}(k=1,2, \ldots, K)$ sub-block in $\hat{s}[n]$. For each sub-block $\hat{s}_{k}[n]$ we computed the following features.

- Mel-frequency cepstral coefficients (MFCCs): MFCCs is widely used in speech processing [18]. It was found to be highly useful for snore analysis [19-22] as well as cough analysis [11]. MFCCs provide a compact representation of the upper airway acoustical properties and allow one to separate contributions from the airway cavity geometry and the source vibration sounds. The MFCCs $\left(\phi_{k}\right)$ of a sub-block $\hat{s}_{k}[n]$ can be computed using (3).

$$
\phi_{k}=\sum_{c=1}^{c} L_{k}(c) \cos \left\{\frac{r(2 c-1) \pi}{2 C}\right\}
$$

Note that in (3), $L_{k}$ is log energy output of $c$ Mel Filter banks $(c=1,2, \ldots, 40)$ of a subblock $\hat{s}_{k}[n]$ and $r$ is the number of cepstral coefficients $(r=0,1, \ldots, 13)$. The MFCCs $\left(\phi_{k}\right)$ of all sub-blocks in $\hat{s}[n]$ can be expressed in a matrix form as:

$$
\phi=\left[\phi_{1}, \phi_{2}, \ldots, \phi_{k}, \ldots, \phi_{K}\right]
$$

- Formant frequency: In speech, Formant frequency shows the characteristics of vocal tract resonances; in snore sound analysis they indicate the resonance of the upper airway. We 
hypothesized that in cough/respiratory sounds, formant may carry resonances of the entire respiratory tract. For instance, wheezing sounds, which originates due to vibrations of the bronchioles of the lung, may contribute higher frequency formants (resonance frequencies) in the cough sounds. We used linear predictive coding (LPC) to estimate the formant frequency. To find the coefficients, LPC uses autocorrelation method of autoregressive (AR) model which can be determined by solving Yule-Walker equations via the LevinsonDurbin recursion [23].

In this work, we computed five formant frequencies per sub-block. The formant frequency of a sub-block $\hat{s}_{k}[n]$ is denoted by $\Re_{k}$. In a matrix notation, we write the formant frequencies $\Re$ of all $K$ sub-blocks of $\hat{s}[n]$ as (5).

$$
\mathfrak{R}=\left[\mathfrak{R}_{1}, \mathfrak{R}_{2}, \ldots, \mathfrak{R}_{k}, \ldots, \mathfrak{R}_{K}\right]
$$

- Zero crossing rates (ZCR): The ZCR, defined as the total time a signal crosses the zero axis, is a simple but useful method to detect the periodic nature of a signal regardless of its magnitude. It may represent the glottis vibrations and can be used to separate voice and unvoiced signal [24]. The ZCR $\left(Z_{k}\right)$ of a sub-block $\hat{s}_{k}[n]$ can be calculated using (6).

$$
Z_{k}=\frac{1}{N-1} \sum_{n=1}^{N-1} H\left\{-\hat{s}_{k}(n) \hat{s}_{k}(n-1)\right\}, \quad 1 \leq n \leq N-1
$$

where $\mathrm{H}\{e\}$ is Heaviside function (its value is 0 for negative argument and 1 for otherwise). The ZCR $(Z)$ of all $K$ sub-blocks of $\hat{s}[n]$ signal is given in (7).

$$
Z=\left[Z_{1}, Z_{2}, \ldots, Z_{k}, \ldots, Z_{K}\right]
$$

- Non-Gaussianity score (NGS): The NGS provides an easy method to quantify the deviation of a given signal from a Gaussian model. In our previous work on snore sound analysis [25], this feature showed a capability to screen obstructive sleep apnea/OSA (OSA snoring tends to produce non-Gaussian distributed sounds). In cough, the non-Gaussianity may arise when 
the glottis suddenly opens during the expulsive phase. The NGS $\left(\psi_{k}\right)$ of a sub-block $\hat{s}_{k}[n]$ can be calculated using (8), where $p$ and $q$ are the normal probability plot of the reference normal data and analyzed data, respectively and $\bar{q}$ represents mean of $q$.

$$
\psi_{k}=1-\left(\frac{\sum_{j=1}^{N}\left(q_{j}-p\right)^{2}}{\sum_{j=1}^{N}\left(q_{j}-\bar{q}\right)^{2}}\right), \quad 1 \leq j \leq N
$$

The NGS $(\psi)$ of all sub-blocks in $\hat{s}[n]$ is denoted in (9).

$$
\psi=\left[\psi_{1}, \psi_{2}, \ldots, \psi_{k}, \ldots, \psi_{K}\right]
$$

- Shannon entropy: Cough sound is a complex signal which represents contributions from various sub-structures of the respiratory tract. Some of these components display pseudoperiodic structures, while others have a random stochastic character. In this work, we computed the Shannon entropy to capture these features. The Shannon entropy $\left(\eta_{k}\right)$ of a subblock $\hat{s}_{k}[n]$ was obtained using definition in (10) [26].

$$
\eta_{k}=-\sum_{n=1}^{N-1}\left(\hat{s}_{k}(n)^{2}\right) \ln \left(\hat{s}_{k}(n)^{2}\right), \quad 1 \leq n \leq N-1
$$

We formed the matrix $\eta$ of all sub-blocks in $\hat{s}[n]$ as follows.

$$
\eta=\left[\eta_{1}, \eta_{2}, \ldots, \eta_{k}, \ldots, \eta_{K}\right]
$$

For each sub-block $\hat{s}_{k}[n]$, compute all the features described in Section 2.4 .2 and form a feature vector $F_{k}=\left[\begin{array}{lllll}\phi & R & Z & \psi & \eta\end{array}\right]^{T}$. We extracted these feature vectors from both Model Development Dataset (MDD) and the Prospective Validation Set (PSD). Let the feature set extracted from the $k^{\text {th }}$ sub-block of MDD be denoted by $F_{k, \mathrm{M}}$ and that from the PSD be denoted $F_{k, \mathrm{P}}$

Next we formed an overall feature matrix based on the feature vectors of sub-blocks as: $G_{M}=\left\{F_{1, M}, F_{2, M}, \ldots, F_{k, M}, \ldots, F_{K, M}\right\}$ and $G_{P}=\left\{F_{1, P}, F_{2, P}, \ldots, F_{k, P}, \ldots, F_{K, P}\right\}$, where $G_{M}$ and $G_{P}$ represent feature matrices for MDD and PSD. 
Cough feature matrix estimated from the set $\mathrm{MDD}, G_{M}$, was then used to train the automatic classifier model to classify sound data in a sub-block $\hat{s}_{k}[n]$ into the classes cough (CG) and non-cough (NC). Details of this process are described in Section 2.4.3. The matrix $G_{P}$ from the set PSD was used for the prospective testing of the trained models.

\subsubsection{Design of automatic classification model}

The automatic cough segmentation method is a two stage process: classification of sound features into cough (CG) and non-cough (NC) classes, and identification of cough events. The description of these processes is given in Section 2.4.3(A) and 2.4.3(B).

(A) Design of neural network model to classify a sub-block $\hat{s}_{k}[n]$ into the cough (CG) and non-cough (NC) classes

In this paper, we investigate the use of an Artificial Neural Network (ANN) as the CG/NC classifier at the sub-block level. We used the ANN inspired by the capability of the human brain to recognize different types of cough sounds regardless of their intensity, duration, or wetness. Moreover, ANN has the advantage of classifying data using non-linear decision boundaries, based on a process of supervised learning with a set of given examples. We compared the performance of the ANN with linear discriminant analysis (LDA) to justify the selection of the non-linear classifier for this work. In contrast to ANN, LDA searches for the linear combination of features [27] that give the best possible separation between cough and non-cough sounds.

In this work, we used the particular form of an ANN known as a Time Delay Neural Network (TDNN) [28] that has been used in speech recognition applications. TDNN is capable of classifying sub-blocks $\hat{s}_{k}[n]$ discounting temporal translations of the input feature set [28].

Our TDNN structure comprised of an input layer $\left(L_{i}\right)$, two hidden layers $\left(L_{h 1}\right.$ and $\left.L_{h 2}\right)$, and an output layer $\left(L_{o}\right)$. The number of neurons in $L_{i}$ depends on the size of input feature vector to ANN which is defined in next paragraph. The number of neurons in $L_{h 1}, L_{h 2}$, and $L_{o}$ were set to 20,10 , and 1 respectively. We used a linear activation function for neurons in $L_{o}$ and sigmoid 
activation functions for neurons in $L_{h 1}$ and $L_{h 2}$ layers. To determine initial weights and bias values, we used the Nguyen-Widrow initialization method [29]. For updating weights during the training process, we employed the resilient back propagation (RPROP) algorithm [30].

We trained the TDNN to classify each sub-block of $\hat{s}[n]$ into CG/NC class. Let $F_{k-2} F_{k-1}$ $F_{k} F_{k+1} F_{k+2}$ represent the feature vectors of sub-blocks $\hat{s}_{k-2}[n], \hat{s}_{k-1}[n], \hat{s}_{k}[n], \hat{s}_{k+1}[n], \hat{s}_{k+2}[n]$, respectively. Let $\mathrm{Q}=\left[\begin{array}{llllll}F_{k-2} & F_{k-1} & F_{k} & F_{k+1} & F_{k+2}\end{array}\right]$ be the input feature vector to ANN formed by concatenating individual feature vector from sub-blocks. Therefore number of neurons in $L_{i}$ of TDNN will be 110 . We used $Q$ as input to the TDNN to classify the $k^{\text {th }}$ sub-block of $\hat{s}_{k}[n]$ into $\mathrm{CG} / \mathrm{NC}$ class. This process was repeated for $k=3,4, \ldots, K-2$ to cover the whole signal $\hat{s}[n]$ represented by $\hat{s}[n]=\left\{\left|\hat{s}_{1}[n]\right|, \ldots,\left|\hat{s}_{k}[n]\right|, \ldots,\left|\hat{s}_{K}[n]\right|\right\}$.

In training and optimizing the TDNN, we used the matrix $G_{M}$ and adopted the leave-one out validation (LOOV) technique. This involves using feature matrices from all the subjects in MDD except one to train the TDNN model, and validate the model using the remaining subject. This process was systematically repeated 10 times such that each subject in MDD was used as the validation data once. This resulted in 10 neural network models.

Let $u_{m}[k], m=1,2, \ldots, 10$, represents the output of the $m^{\text {th }}$ TDNN. In Section 2.4.3 (B), we describe the method to identify the beginning and the end of a cough event using $u_{m}[k]$.

\section{(B) Identification of the beginning and the end of cough events}

The identification of the beginning and the end of cough events from the output of TDNN models $u_{m}[k]$ was carried out by following steps (S1) - (S3):

(S1). Smoothing process - Pass $u_{m}[k]$ through a moving average filter with the tap length $\beta$. Let the output of this filter be $\tilde{u}_{m}[k]$.

(S2). Thresholding - Apply a threshold value $\lambda$ to $\tilde{u}_{m}[k]$. The sub-blocks with $\tilde{u}_{m}[k]$ above the threshold $\lambda$ were assigned the decision $\gamma=1$ otherwise $\gamma=0$. The sub-blocks with value $\gamma=1$ are candidate cough event sub-blocks. 
(S3). Cough event identification - A cough event is identified if $\rho$ number of consecutive sub-blocks are candidate sub-blocks (i.e. $\gamma=1$ ) and if following conditions are satisfied:

Condition 1: $\tau_{\min } \leq \tau_{\rho} \leq \tau_{\max }$, where $\tau_{\rho}$ is the total time duration of $\rho$ consecutive subblocks with $\gamma=1$. $\tau_{\min }$ and $\tau_{\max }$ are the minimum and maximum cough sound durations computed from the cough events in MDD.

Condition 2: $\delta_{\rho}>\delta$, where $\delta \rho$ is the root mean square (RMS) value of $\rho$ consecutive sub-blocks in $\tilde{u}_{m}[k]$ with $\gamma=1$ and $\delta$ is the threshold root mean square value.

Both Conditions 1 and 2 were adopted to improve the specificity of the automatic classifier by rejecting those episodes which are falsely identified as probable cough episodes after S2. While Condition 1 checks for the duration of events, removing lengthy episodes which are more likely to be non-cough sounds, Condition 2 checks for the RMS value of TDNN output. Cough events in MDD indicated that RMS value of TDNN output for cough sounds were higher than that of non-cough sounds.

All the parameters including $\beta, \lambda$ and $\delta$ were optimized using data in MDD for maximizing the classifier performance. The steps for optimizing these parameters are as follows:

(P1). Optimizing $\beta$ - set parameter $\lambda$ and $\delta$ at certain values then vary $\beta$ from 1 to 30 . Select the optimum $\beta$ values at the maximum performance in the validation set.

(P2). Optimizing $\lambda$ - use the optimized $\beta$ and $\delta$ values from step (P1), and then vary $\lambda$ from 0.001 to 1 . Select the optimum $\lambda$ at the maximum performance in the validation set.

(P3). Optimizing $\delta$ - use the optimized $\beta$ and $\lambda$ values from steps (P1) and (P2), and then vary $\delta$ from 0.001 to 1 . Select the optimum $\delta$ at the maximum performance in the 
validation set.

To evaluate the performance of the proposed method, performance measures such as sensitivity, specificity, accuracy, and Cohen's Kappa statistic were computed. The performances were computed both at the sub-block level as well as the cough event level by comparing the output of the algorithm with the reference scoring from the human observer.

From the ten TDNN models, we selected the model that gave us the best performance as obtained in our LOOV validation technique on MDD. Let $\aleph$ represent the selected TDNN model with $\beta_{s}, \lambda_{s}, \delta_{s}$ as its corresponding parameters. The implementation of $\aleph$ on the prospective dataset is described in the following section.

\subsubsection{Testing the selected model $\aleph$ on the prospective dataset.}

The process of cough segmentation in PSD is as follows. Let $G_{P}$ be a feature matrix computed using sound data from a subject in dataset PSD, following the process described in Section 2.4.1 - 2.4.2. Apply the model $\aleph$ to $G_{P}$ and automatically classify the cough sounds into classes $\mathrm{CG} / \mathrm{NC}$ at the sub-block level; identify cough events following the steps given in Section 2.4.3. Repeat this for all the patients $\left(D_{2}=14\right)$ in PSD. Compare the results of automatic segmentation with that of manual segmentation and evaluate the performance, at sub-block as well as event levels. In Section 3.5, we describe the performance of $\aleph$ in segmenting coughs from the prospective study data set (PSD).

\section{RESULTS}

\subsection{Dataset}

In this work, we included cough sound data from 24 subjects (13 males and 11 females). The age of the subjects spanned from 3 months to 71 months (14 subjects less than 12 months and 10 subjects greater than 12 months). After the clinical evaluation and laboratory tests, 18 
were diagnosed with pneumonia, one each with rhinopharyngitis, nasopharyngitis, tonsillopharyngitis, pulmonary hypertension, bronchiectasis and bronchitis.

Training dataset MDD has 656 cough and 2297 non-cough events spread over a 15minute period. Each subject contributed, on the average, 66 coughs to the set MDD. The minimum length of a cough event $\left(\tau_{\min }\right)$ was $180 \mathrm{~ms}$ and the maximum $\left(\tau_{\max }\right)$ was $720 \mathrm{~ms}$.

Prospective dataset PSD has a total sound data of 840 minutes duration from $D_{2}=14$ subjects. In this dataset, there were 1434 coughs and 40144 non-cough sounds. The coughs have minimum and maximum length of $160 \mathrm{~ms}$ and $800 \mathrm{~ms}$, respectively. On the average, there were 96 coughs in one hour of sound data from each subject. We illustrate the length distribution
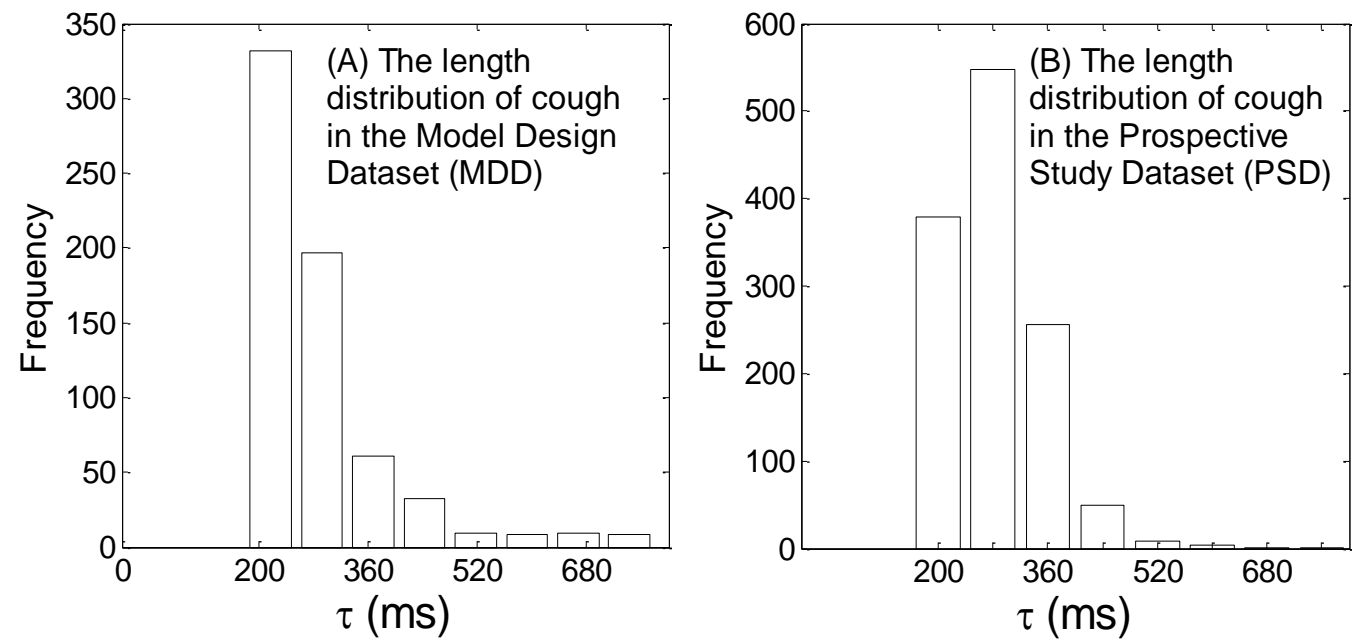

Figure 3. Histogram of the cough duration in (A) MDD and (B) PSD. The minimum $\left(\tau_{\min }\right)$, maximum $\left(\tau_{\max }\right)$, and mean length of cough were $180 \mathrm{~ms}, 720 \mathrm{~ms}$ and $283 \mathrm{~ms}$, respectively, in MDD and $160 \mathrm{~ms}, 800 \mathrm{~ms}$ and $291 \mathrm{~ms}$, respectively, in PSD.

of coughs in Fig 3.

\subsection{Noise reduction}

Figure 4 illustrates the effect of HPF and PSS filters described in Section 2.4.1 to remove the noise components $b_{1}[n]$ and $b_{2}[n]$ from the raw signal $s[n]$. Top panel of Fig 4 shows: (A) sample raw cough signal, (B) signal after HPF, (C) signal after HPF + PSS and (D) (E) and (F) in bottom panel of Fig 4 shows power spectrum of respective signals in (A), (B) and (C). From the power spectra (Fig 4(D)) of the raw signal in Fig 4(A), it can be seen that the raw signal 

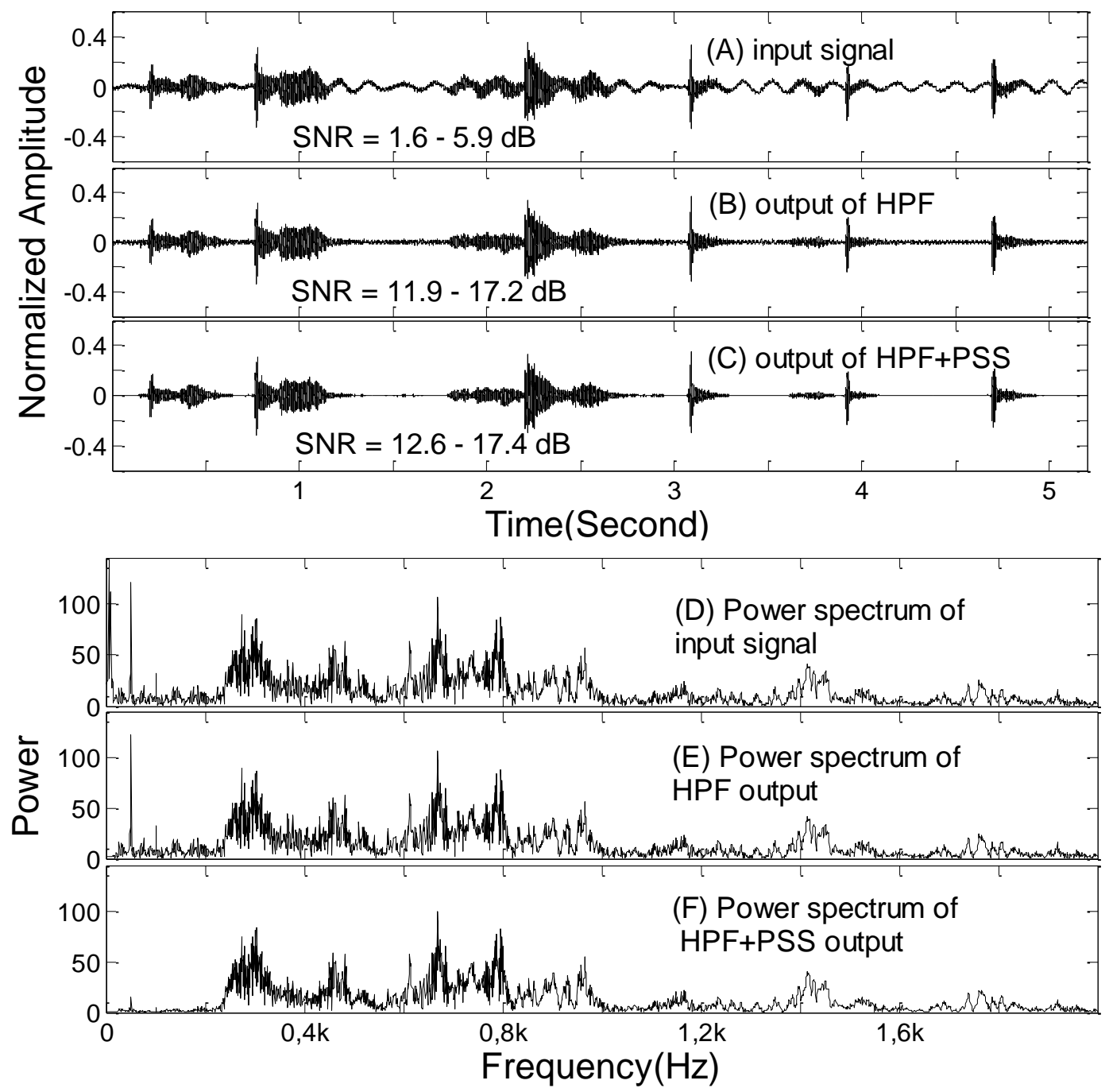

Figure 4. Illustration of the noise reduction process in time domain (A, B and C) and frequency domain $(\mathrm{D}, \mathrm{E}$ and $\mathrm{F}) . \mathrm{HPF}=$ High Pass Filter and PSS = Power Spectral Subtraction filter. (A) Sample raw cough signal, (B) signal after HPF, (C) sample cough signal after HPF + PSS (D) power spectrum of raw cough signal, (E) power spectrum of signal after HPF, (F) power spectrum of signal after HPF + PSS. SNR range indicates the lowest and the highest SNR of the cough segment in the displayed audio signal. It is clear that with the filtering improves SNR of the cough signal considerably.

$s[n]$ has low frequency interference $(<10 \mathrm{~Hz})$ as well as $50 \mathrm{~Hz}$ interference. We defined the signal to noise ratio $(\mathrm{SNR})$ as: $\mathrm{SNR}=20 \log _{10}(\mid$ Signal Magnitude $/ /$ Noise Magnitude $\mid) \mathrm{dB}$. The SNR of the raw recording $s[n]$ in this example, was very low $(<6 \mathrm{~dB})$.

Figures 4(B) and Fig 4(C) show the output signal $\hat{s}[n]$ after the application of HPF and PSS filters. Their power spectra are shown in Fig 4(E) and Fig 4(F), respectively. According 
Table 1 Performances of TDNN classifier compared with the LDA classifier on training dataset MDD using four different sub-block sizes. Statistics provided in the table are mean \pm standard deviation. Tr, Va, Sens, and Spec respectively indicate training set, validation set, sensitivity and specificity.

\begin{tabular}{|c|c|c|c|c|c|c|}
\hline \multirow{2}{*}{\multicolumn{3}{|c|}{ Classifiers }} & \multicolumn{4}{|c|}{ Sub-block size } \\
\hline & & & $10 \mathrm{~ms}$ & $20 \mathrm{~ms}$ & $40 \mathrm{~ms}$ & $60 \mathrm{~ms}$ \\
\hline \multirow{4}{*}{ LDA } & \multirow{2}{*}{$\operatorname{Tr}$} & Sens & $77.18 \pm 0.6$ & $78.47 \pm 0.5$ & $79.18 \pm 0.5$ & $59.83 \pm 0.4$ \\
\hline & & Spec & $78.31 \pm 0.6$ & $78.71 \pm 0.5$ & $75.31 \pm 0.6$ & $70.95 \pm 1.1$ \\
\hline & \multirow{2}{*}{$\mathrm{Va}$} & Sens & $76.14 \pm 4.6$ & $77.49 \pm 5.4$ & $78.62 \pm 6.1$ & $59.78 \pm 8.0$ \\
\hline & & Spec & $74.24 \pm 8.0$ & $74.75 \pm 7.5$ & $71.15 \pm 7.7$ & $61.26 \pm 15.2$ \\
\hline \multirow{4}{*}{ TDNN } & \multirow{2}{*}{$\operatorname{Tr}$} & Sens & $90.56 \pm 1.8$ & $92.92 \pm 2.1$ & $92.92 \pm 1.9$ & $74.72 \pm 2.6$ \\
\hline & & Spec & $90.57 \pm 1.8$ & $92.92 \pm 2.1$ & $92.93 \pm 1.9$ & $74.72 \pm 2.6$ \\
\hline & \multirow{2}{*}{$\mathrm{Va}$} & Sens & $84.09 \pm 3.4$ & $87.16 \pm 3.1$ & $87.84 \pm 2.7$ & $67.49 \pm 3.3$ \\
\hline & & Spec & $84.11 \pm 3.4$ & $87.17 \pm 3.1$ & $87.91 \pm 2.7$ & $67.56 \pm 3.3$ \\
\hline
\end{tabular}

to these figures the designed filters reduced the low frequency interference $(<10 \mathrm{~Hz})$ and $50 \mathrm{~Hz}$ interference significantly. The SNR of the filtered signal $\hat{s}[n]$ increased to $>17 \mathrm{~dB}$.

\subsection{Parameter optimization of the classifier model using the set MDD}

Following the process described in Section 2.4.2 and using the data from MDD, a cough feature matrix $G_{M}$ was created. $G_{M}$ was then used to train the automatic classifier models (TimeDelay Neural Network, TDNN and Linear Discriminant Analysis, LDA) to classify sound data in sub-blocks $\hat{s}_{k}[n]$ into classes cough (CG) and non-cough (NC) (Section 2.4.3).

We varied the length of sub-blocks to optimize the classification performance (sensitivity, specificity) of $m=10 \mathrm{TDNN}$ and LDA models. The performances of TDNN compared with LDA are shown in Table 1. From the table it can be seen that TDNN outperforms LDA in 
Table 2 Performances of TDNN on the training dataset MDD, using different combination of features. Statistics provided in the table are mean \pm standard deviation. $R=$ Formant frequency, $H=$ Shannon entropy, $Z=$ Zero Crossing Rate, $\psi=$ Non-Gaussianity Score, and $\phi=$ MFCCs. Tr, Va, Sens, and Spec, respectively indicate training set, validation set, sensitivity and specificity.

\begin{tabular}{ccccccc}
\hline & & \multicolumn{5}{c}{ Features } \\
\cline { 3 - 7 } & & $\Re$ & $\Re, H$ & $\Re, H, Z$ & $\Re, H, Z, \psi$ & $\Re, H, Z, \psi, \varnothing$ \\
\hline \multirow{2}{*}{$\operatorname{Tr}$} & Sens & $71.68 \pm 3.1$ & $73.29 \pm 3.2$ & $79.52 \pm 2.7$ & $81.09 \pm 2.4$ & $92.92 \pm 2.1$ \\
& Spec & $71.68 \pm 3.1$ & $73.29 \pm 3.2$ & $79.52 \pm 2.7$ & $81.10 \pm 2.4$ & $92.92 \pm 2.1$ \\
\hline & Sens & $68.47 \pm 4.4$ & $70.21 \pm 5.2$ & $77.32 \pm 5.3$ & $78.89 \pm 4.3$ & $87.16 \pm 3.1$ \\
Va & & & & & & \\
& Spec & $68.48 \pm 4.4$ & $70.24 \pm 5.2$ & $77.35 \pm 5.3$ & $78.92 \pm 4.3$ & $87.17 \pm 3.1$ \\
\hline
\end{tabular}

classifying cough and non-cough sounds in all sub-block sizes. As such we used TDNN for the rest of this work.

Table 1 also shows that the sub-block size ranging from 20 - 40 ms gave a similar performance in TDNN. Therefore we set sub-block size $=20 \mathrm{~ms}$ in Section 2.4.2. The reason behind this choice is that a shorter sub-block can give a better time resolution. Table 2 shows TDNN model performance when different combinations of feature sets were used in the training. From Table 2 it can be seen that when we used all features to train TDNN, it gave the best performance both in training and leave one out validation (LOOV). For this reason, results reported in this paper use TDNN models trained with all these features.

Parameters $\beta, \lambda$ and $\delta$ were optimized to achieve the best classification performance following the process described in Section 2.4.4. Figure 5 shows parameter optimization results for $\beta, \lambda$ and $\delta$. According to Fig 5(A), at low $\beta$ values, the classification sensitivity is high. It starts decreasing as $\beta$ increases above 10 . No significant variation in validation specificity can be seen with $\beta$ value. In case of the parameter $\lambda$ (Fig 5(B)), the validation sensitivity increases 


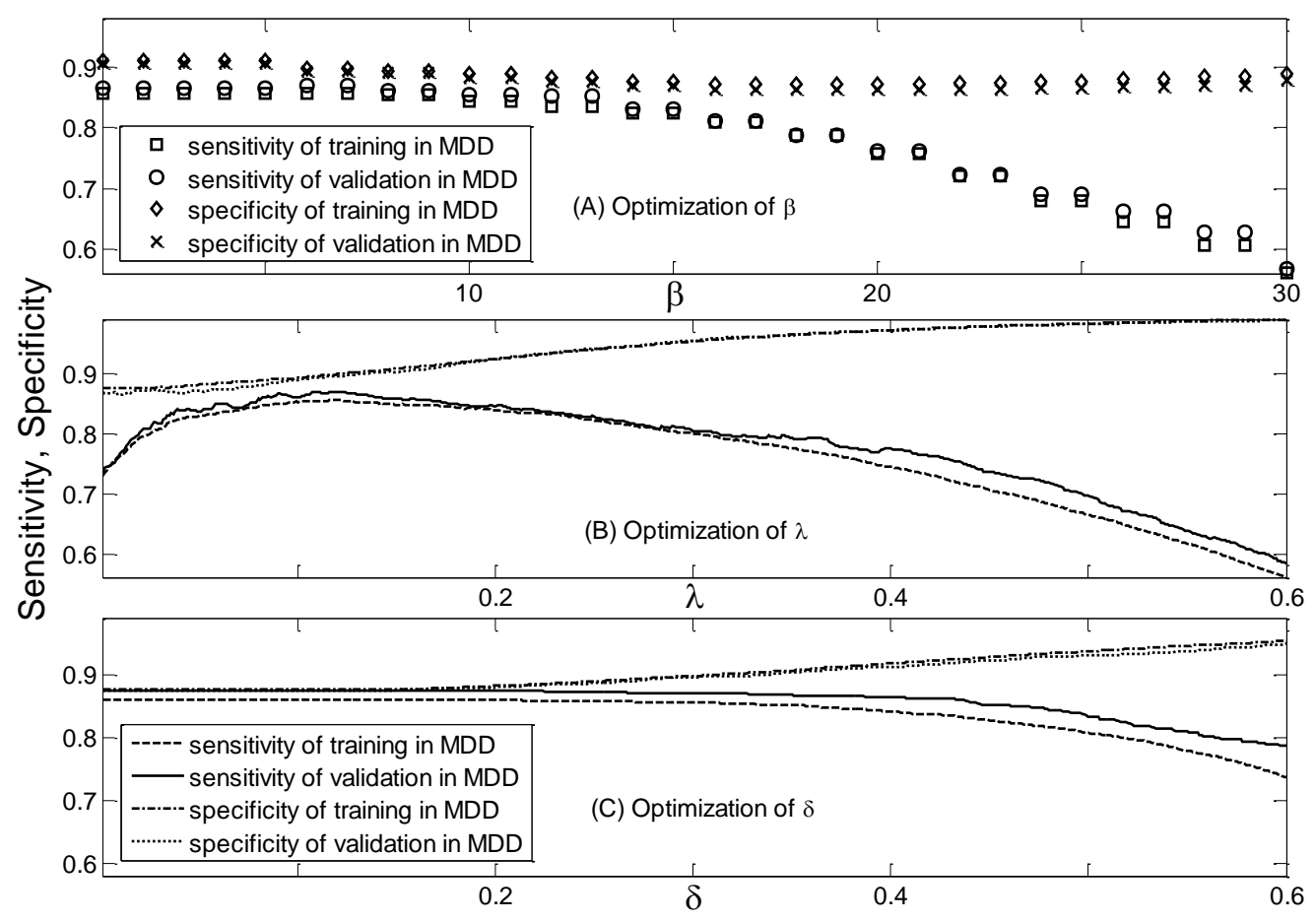

Figure 5. Illustration of classifier parameters optimization in model design dataset (MDD).

(A) Optimization of moving average filter tap length $\beta(\lambda=0.1$ and $\delta=0.3)$, (B) Optimization of threshold value $\lambda(\beta=9$ and $\delta=0.3)$ and (C) Optimization of the root mean square threshold $\delta(\beta=9$ and $\lambda=0.116)$.

with $\lambda$ reaching the peak value at $\lambda=0.116$ and then starts decreasing. On the other hand, the validation specificity shows a consistent increase with an increase in $\lambda$. For the parameter $\delta$, validation sensitivity shows no variation until $\delta=0.229$ and then starts decreasing. Contrary to this, validation specificity starts increasing after $\delta=0.229$. Using these curves we set $\beta_{s}=9, \lambda_{s}$ $=0.116$ and $\delta_{s}=0.327$.

\subsection{Segmentation results on training/validation dataset MDD}

In this section, we illustrate the cough segmentation process and present the segmentation results on dataset MDD. The segmentation results are presented at the sub-block level as well as the cough event level.

We illustrate a typical cough sound segment from MDD and the output of automatic segmentation at different stages of processing in Fig 6. Figure 6(A) shows the filtered signal 


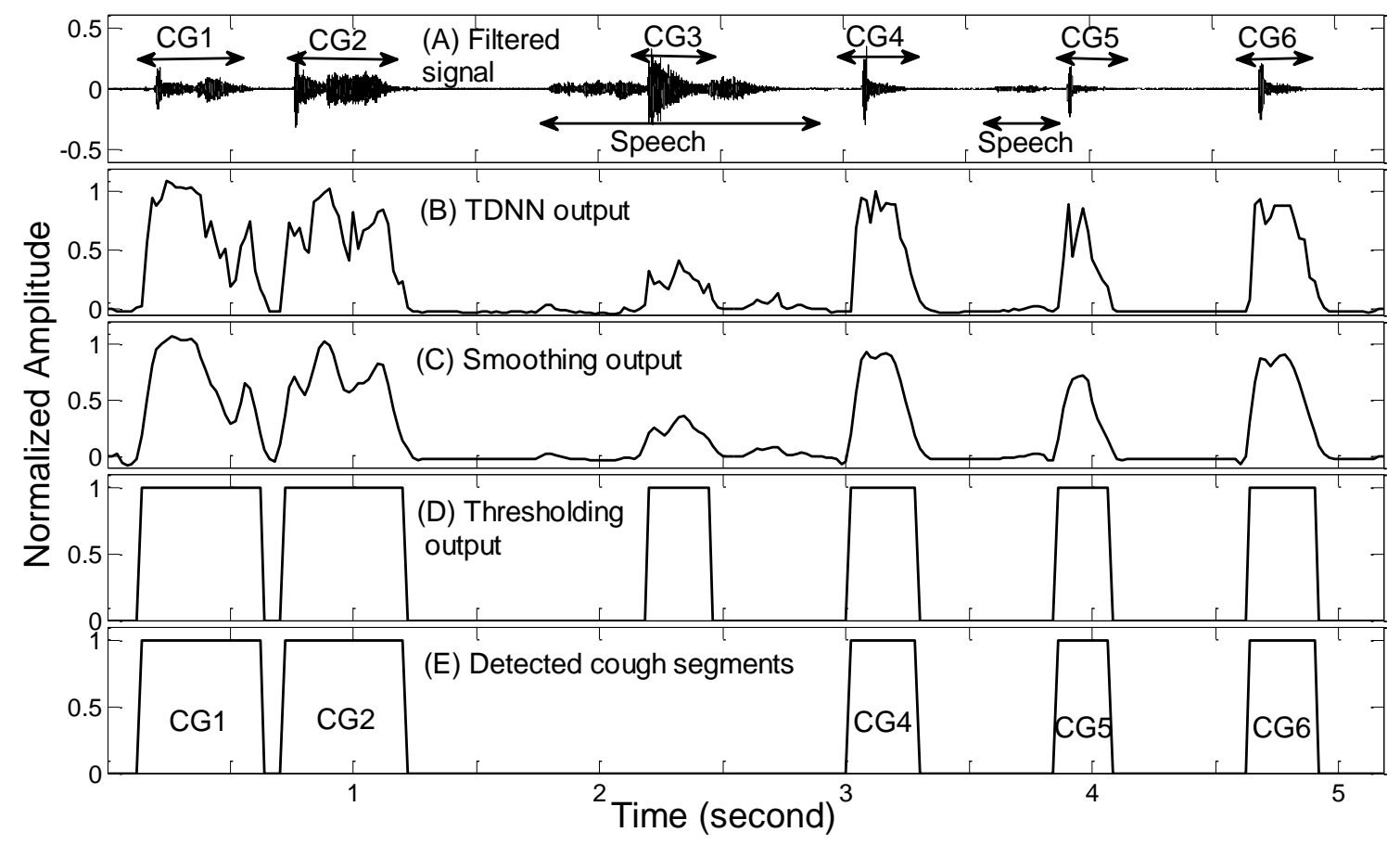

Figure 6. Illustration of cough segment identification from the model design dataset MDD. The cough sounds are indicated by CG1 - CG6. (A) Output of noise reduction process, (B) Output of TDNN, (C) Output of moving average filter (step (S1) in Section 2.4.3), (D) Output of thresholding (step (S2) in Section 2.4.4), (E) Cough segments identified after step (S3) in Section 2.4.4).

$\hat{s}[n]$ and Fig 6(B) shows the output signal $u_{m}[k]$. According to Fig 6(B), $u_{m}[k]$ is high (very close to 1) during cough events (CG1, CG2, CG4, CG5, and CG6). Even though the intensity of CG5 is low (Fig 6(A)), $u_{m}[k]$ is still high. In Fig 6(A), CG3 is a cough event mixed with a speech signal, and in that case $u_{m}[k]$ has a low value.

To determine the beginning and the end of cough segments from $u_{m}[k]$, we followed the process described in Section 2.4.3 steps $(S 1)-(S 3)$. Figures 6(C) - Fig 6(E) show the outputs of those steps. Figure $6(\mathrm{C})$ shows the output of the smoothing process $\tilde{u}_{m}[k]$. According to Fig 6(C), smoothing process improves the shape of the signal by reducing the spurious transitions of $u_{m}[k]$, as in the case of CG5.

Figure 6(D) shows the candidate cough segments after thresholding. At step (S3) of Section 2.4.3, only the candidate segments fulfilling criteria $\left(\tau_{\min } \leq \tau_{\rho} \leq \tau_{\max }\right.$ and $\delta_{\rho}>\delta$ ) were 
Table 3: The performance of the algorithm on the training dataset (MDD) and prospective study dataset (PSD) using the optimized parameters ( $\delta_{s}=0.327$, when $\left.\beta_{s}=9, \rho_{s}=0.116\right)$. Statistics provided in the table are mean \pm standard deviation.

\section{Training results for 10 TDNN models on dataset MDD}

(A) Performance computed at sub-block level

\begin{tabular}{lcccc}
\hline & Accuracy & Sensitivity & Specificity & Kappa \\
\hline Training & $89.4 \pm 1.0$ & $85.5 \pm 2.3$ & $90.5 \pm 0.8$ & $0.71 \pm 0.03$ \\
Validation & $89.4 \pm 3.3$ & $84.6 \pm 8.6$ & $90.4 \pm 3.9$ & $0.71 \pm 0.06$ \\
\hline
\end{tabular}

(B) Performance computed at event level (overlap > 50\%)

\begin{tabular}{lcccc}
\hline & Accuracy & Sensitivity & Specificity & Kappa \\
\hline Training & $93.6 \pm 1.2$ & $91.3 \pm 1.9$ & $94.8 \pm 1.2$ & $0.82 \pm 0.03$ \\
Validation & $93.9 \pm 2.0$ & $89.8 \pm 7.0$ & $94.8 \pm 1.7$ & $0.82 \pm 0.06$ \\
\hline
\end{tabular}

Testing results using selected TDNN model $\aleph\left(\delta, \beta_{s}, \rho_{s}\right)$ on dataset PSD

(C) Performance computed at sub-block level

\begin{tabular}{lcccc}
\hline & Accuracy & Sensitivity & Specificity & Kappa \\
\hline Testing & $99.1 \pm 0.5$ & $83.7 \pm 8.6$ & $99.3 \pm 0.4$ & $0.60 \pm 0.1$ \\
\hline & (D) Performance computed at event level (overlap > 50\%) \\
\hline Testing & Accuracy & Sensitivity & Specificity & Kappa \\
\hline
\end{tabular}

classified as the Cough Segments. Figure 7(E) shows the output after step (S3). According to Fig 6(E), segments CG1, CG2, CG4, CG5, and CG6 were correctly identified as cough events. The event CG3, which has speech segments, was not identified as a cough event. These results indicate that the proposed automated algorithm is capable of rejecting cough events corrupted with speech sounds and is robust against the variation of cough sound intensities.

In Table 3(A) and Table 3(B), we present segmentation results obtained from the ten TDNN models in our leave-one-out validation (LOOV). Table 3(A) shows the segmentation performance computed at the sub-block level. At the sub-block level, the algorithm achieved a sensitivity and specificity of $85.5 \pm 2.3 \%$ and $90.5 \pm 0.8$ in the TDNN training process. The 
trained model resulted in a sensitivity and specificity of $84.6 \pm 8.6 \%$ and $90.4 \pm 3.9 \%$ respectively in the LOOV process.

Cohen's Kappa (K) statistic is widely used in situations where the agreement between two techniques should be compared. The guidelines for interpreting $\mathrm{K}$ are, $\mathrm{K}<0$ : less than chance agreement, $0.01-0.20$ : slight agreement; $0.21-0.40$ : fair agreement, $0.41-0.60$ : moderate agreement, $0.61-0.80$ : substantial agreement, $0.81-1$ : almost perfect agreement. The $\mathrm{K}$ value between automatic classification and manual segmentation during LOOV was $0.71 \pm 0.06$ indicating a substantial agreement.

Table 3(B) shows the performance of the automated algorithm in identifying cough events. For the work of this paper, a true positive was detected if event identified by automatic classifier had at-least $50 \%$ overlap with manual scorer. The LOOV sensitivity and specificity in identifying cough events were $89.8 \pm 7.0 \%$ and $94.8 \pm 1.7 \%$ respectively. The validation Kappa agreement between automated algorithm and scorer was $0.82 \pm 0.06 \%$, which signifies almost perfect agreement.

\subsection{Segmentation results on prospective study dataset PSD}

Following the process in Section 2.4.4, the TDNN model $\aleph$ (with all the parameters fixed) was constructed and tested on the set PSD. Table 3(C) shows the performance of $\aleph$ at the subblock level. It achieved an accuracy, sensitivity and specificity of $99.1 \pm 0.5 \%, 83.7 \pm 8.6 \%$, and $99.3 \pm 0.4 \%$, respectively. The Kappa agreement between $\aleph$ and manual scoring was $0.61 \pm 0.13$ (substantial agreement). Table 3(D) presents the performance of $\aleph$ in identifying cough events (cough-event level) in PSD. Automated algorithm achieved $97.3 \pm 1.1 \%$ of accuracy, $92.8 \pm 6.8 \%$ of sensitivity, $97.5 \pm 1.1 \%$ of specificity, with Kappa value of $0.65 \pm 0.1$ in identifying cough events. 
Table 4: Performance of the selected TDNN model $M_{s}\left(\delta_{s}, \beta_{s}, \rho_{s}\right)$ on the Prospective Study Dataset (PSD).

\begin{tabular}{lccc}
\hline & $\begin{array}{c}\text { Less than 12 } \\
\text { months }\end{array}$ & 12 to 36 months & 36 to 72 months \\
\hline Accuracy & $97.0 \pm 1.4$ & $97.1 \pm 0.2$ & $97.7 \pm 0.3$ \\
Sensitivity & $91.4 \pm 8.1$ & $94.6 \pm 4.7$ & $92.2 \pm 7.8$ \\
Specificity & $97.3 \pm 1.4$ & $97.4 \pm 0.6$ & $98.3 \pm 1.0$ \\
\hline
\end{tabular}

According to Table 3, the performance of the automated algorithm is better on PSD than on MDD. The major reason for this is the way MDD and PSD have been designed, as described in Section 2.2. MDD (training set) consisted of manually picked sounds, with a target of presenting the TDNN with the range of sound episodes observable in a real recording. To make the learning process robust, in MDD we purposely included even difficult examples such as low SNR data and low intensity coughs. In the case of PSD, we used the actual recorded data streams, where such difficult events naturally occur at a much lower rate. Even though this strategy led to a slightly lower performance on the training set, it also led to a better generalization capability, which helped the $\aleph$ to produce a better outcome on PSD.

Table 4 presents the segmentation results in three age groups (less than 12 months, from 12 to 36 months, and from 36 to 65 months). From Table 4, it can be seen that the accuracy and specificity of the automated algorithm is similar across all age groups. The sensitivity in the age group 12-36 months is about $2 \%$ higher compared to the other age groups. The results show that our automated algorithm is not significantly affected by the age variations.

In Fig 7, we further illustrate the performance of the proposed algorithm on sound recordings with different SNRs and a range of cough waveforms. All the sound segments in Fig 7 were taken from the prospective dataset. In Fig 7(A) and Fig 7(B), in spite of cough intensity 

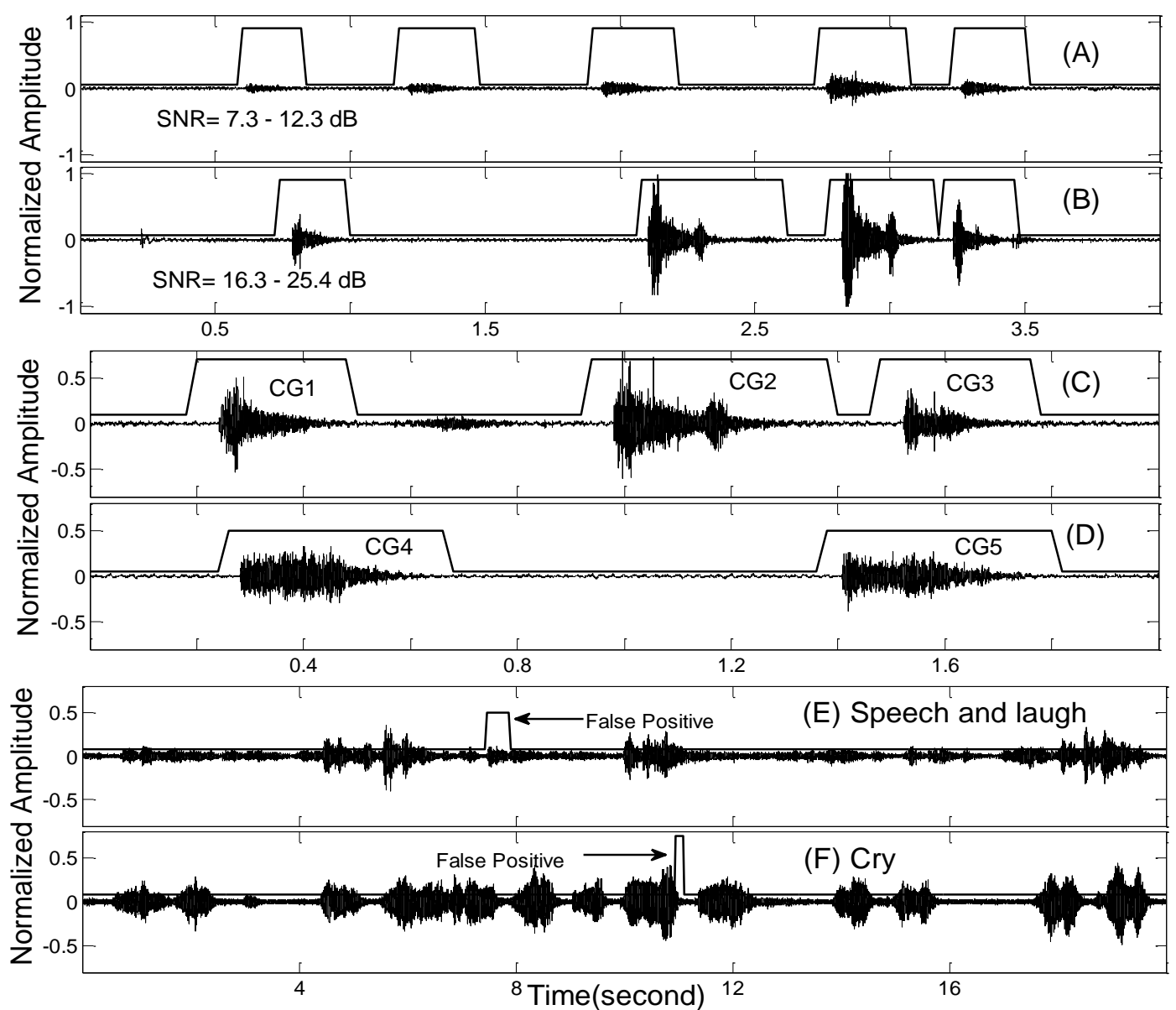

Figure 7. Illustration of cough segment identification in the prospective study dataset (PSD) using the several types of coughs. It can be seen that the proposed algorithm is robust against (i) SNR variation (graphs in (A) and (B)), (ii) waveform-shapes variations (graphs in (C) and (D)), (iii) in rejecting non-cough sounds (graphs in (E) and F)).

varying significantly, the proposed algorithm is able to identify all the cough events. This signifies that the algorithm performance is robust against intensity variations. The waveformshapes (e.g. the number of humps) of cough sounds can vary widely, especially in disease conditions. In order to test whether the algorithm is sensitive to this change, we tested it on two sound segments consisting of coughs with different wave-shapes (see Fig 7(C) and Fig 7(D)). It can be seen from these figures, algorithm identifies the entire cough segment irrespective of their wave-shape. Figure 7(E) and Fig 7(F) show the performance of the algorithm on a typical sound segment consisting of speech, laugh and cries. This result shows the robustness of the algorithm in discounting non-cough sounds regardless of their intensity. 


\section{DISCUSSION}

In this paper, we propose an automated method to identify cough events from pediatric sound recordings acquired with non-contact microphones in the natural environment of a respiratory ward. The subject cohort for our study was children aged 3-71 months having respiratory diseases such as pneumonia, bronchitis and nasopharyngitis. Working on sound recordings from 24 pediatric subjects, we showed that our method was capable of identifying cough events with a sensitivity and specificity of $>90 \%$.

To the best of our knowledge, our work is the first ever attempt to identify cough events from pediatric sound recordings. It is well known that the airway anatomy of children are quite different from those of adults. Cough sounds from the two groups too have different characteristics [31].

The vast majority of subjects in our dataset had pneumonia. The nature of cough changes due to pneumonia. Coughs in pneumonia tend to become shorter and smaller in magnitude. The signal to noise ratio tends to be weaker. Conventional detection methods that depend on features such as gradients [15] and the number of humps of cough waveforms [16] will not be appropriate in detecting pneumonia coughs due to the sharp decrease of the gradients and the deteriorations of hump structures. Our method is novel in that it does not rely on intensity features alone, does not depend on the number of humps, or needs calibration every time it is used. All of these features make our work unique.

Another novelty of our paper is that it analyzed spontaneous coughs from children whereas existing methods $[10,32]$ reported results on voluntary coughs (of adults). Spontaneous coughs contain a wide range of natural variety in terms of cough sound intensity, duration and waveform shape, which cannot be expected in a voluntary cough that would be consciously or subconsciously controlled by an adult subject. Furthermore, our spontaneous (and continuous) sound recordings capture other undesirable sound events such as toy noise, 
crying sounds and adult conversations. Our algorithms assume total naivete to the time of occurrence of any desirable sound event, whereas voluntary recording enables a reasonable control of undesirable sounds and the time of occurrence of desirable events. These differences make spontaneous coughs much more difficult to detect in a continuous sound stream.

We designed and tested our algorithm on a data recorded using non-contact bedside microphones. Previous studies have used body-contact sensors to record sounds. While contact sensors may provide an easier signal to work with, they suffer from the need for a physical sensor contact with the patient. Contact sensors are inconvenient to use on pediatric subjects. They also cause a risk of cross-infection among pneumonia patients. These differences in measurement protocols make our results difficult to compare with those of previous studies.

One of the possible limitations of the present study is the bias of the cohort towards pneumonia patients; $75 \%$ of the children in our dataset had pneumonia. We must test this algorithm on a large dataset with a wider range of respiratory diseases to ensure its robustness.

The results presented in this paper may be further improved by systematically controlling the recording environment when possible. In this paper, we used a recording system that comprised of a laptop computer, microphones, and an analog to digital converter within a hospital setting. Further work is needed to study the potential effects of the recording system and background noise (outside a hospital setting) in the performance of the algorithm. Currently we are working on implementing our technology on smart phones and/or a low-cost sound recorder.

\section{CONCLUSION}

The cough segmentation method proposed in this paper achieved an accuracy, sensitivity, and specificity of $97.3 \%, 92.8 \%$ and $97.5 \%$, respectively on a prospective dataset. The Kappa agreement between our method and the human observer was 0.65 (substantial agreement). Based on these results, we conclude that our method has the potential to be used in automated 
cough logger applications. More importantly, it is possible to use it as the front-end of automatic cough analysis systems $[6,7,33]$.

\section{ACKNOWLEDGEMENT}

This work was supported by the Bill \& Melinda Gates Foundation, USA, under its Grand Challenges in Global Health Explorations Grant program (Grant ID\# OPP1008199 GCE to Abeyratne). 


\section{REFERENCE}

[1] A. J. Hotaling and G. T. Moynihan, "Cough," in Pediatirc Otolaryngology. vol. 2, ed Philadelphia: Saunders, 2003, pp. 1395-1404.

[2] R. E. Black, S. Cousens, H. L. Johnson, J. E. Lawn, I. Rudan, D. G. Bassani, P. Jha, H. Campbell, C. F. Walker, R. Cibulskis, T. Eisele, L. Liu, and C. Mathers, "Global, regional, and national causes of child mortality in 2008: a systematic analysis," The Lancet, vol. 375, pp. 1969-1987, 2010.

[3] Rudan I., Boschi-Pinto C., Biloglav Z., Mulholland K., and Campbell H., "Epidemiology and etiology of childhood pneumonia," Bulletin of the World Health Organization, vol. 86, pp. 408-416, 2008.

[4] World Health Organization, "WHO-recommended standard for surveillance of selectedpreventable diseases," Geneva WHO/V\&B/0301, 2003.

[5] K. F. Chung, "Measurement of cough," Respiratory Physiology \& Neurobiology, vol. 152, pp. 329-339, 2006.

[6] U. R. Abeyratne, Swarnkar V., Setyati A., and Triasih R., "Cough Sound Analysis Can Rapidly Diagnose Childhood Penumonia," Ann Biomed Eng, 2013.

[7] V. Swarnkar, U. R. Abeyratne, Chang A. B., Y. A. Amrulloh, A. Setyati, and R. Triasih, "Automatic Identification of Wet and Dry Cough in Pediatric Patients with Respiratory Diseases," Ann Biomed Eng, 2013.

[8] S. Barry, A. Dane, A. Morice, and A. Walmsley, "The automatic recognition and counting of cough," Cough, vol. 2, p. 8, 2006.

[9] M. A. Coyle, D. B. Keenan, L. S. Henderson, M. L. Watkins, B. K. Haumann, D. W. Mayleben, and M. G. Wilson, "Evaluation of an ambulatory system for the quantification of cough frequency in patients with chronic obstructive pulmonary disease," Cough, vol. 1, p. 3, 2005.

[10] E. Vizel, M. Yigla, Y. Goryachev, E. Dekel, V. Felis, H. Levi, I. Kroin, S. Godfrey, and N. Gavriely, "Validation of an ambulatory cough detection and counting application using voluntary cough under different conditions," Cough, vol. 6, p. 3, 2010.

[11] S. Matos, S. S. Birring, I. D. Pavord, and D. H. Evans, "An Automated System for 24$\mathrm{h}$ Monitoring of Cough Frequency: The Leicester Cough Monitor," Biomedical Engineering, IEEE Transactions on, vol. 54, pp. 1472-1479, 2007.

[12] K. McGuinness, J. Morris, J. Lowe, A. Woodcock, and J. A. Smith, "Automated cough detection: a novel approach [abstract]," Am J Resp Crit Care Med, p. 175: A381, 2007.

[13] A. A. Woodcock, J. A. Smith, and K. McGuinnes, "Method for generating output data," United States Patent 7761302, 2010.

[14] S. Matos, S. S. Birring, I. D. Pavord, and H. Evans, "Detection of cough signals in continuous audio recordings using hidden Markov models," Biomedical Engineering, IEEE Transactions on, vol. 53, pp. 1078-1083, 2006.

[15] M. Coyle, D. B. Keenan, A. Derchak, M. Sackner, F. Wilhelm, K. Gilroy, M. Gummels, D. M. Inma, P. Kennedy, M. Mitchnick, and A. Behar, "Systems and methods for respiratory event detection," United States Patent 7267652, 2007.

[16] Gavriely N., "Phonopneumograph system," United States Patent 6168568, 2001.

[17] R. Martin, "Noise power spectral density estimation based on optimal smoothing and minimum statistics," Speech and Audio Processing, IEEE Transactions on, vol. 9, pp. 504-512, 2001.

[18] S. Greenberg, W. A. Ainsworth, and R. R. Fay, Speech processing in auditory system. New York: Springer, 2004. 
[19] W. D. Duckitt, S. K. Tuomi, and T. R. Niesler, "Automatic detection, segmentation and assessment of snoring from ambient acoustic data," Physiological Measurement, vol. 27, p. 1047, 2006.

[20] E. Goldshtein, A. Tarasiuk, and Y. Zigel, "Automatic Detection of Obstructive Sleep Apnea Using Speech Signals," Biomedical Engineering, IEEE Transactions on, vol. 58, pp. 1373-1382, 2011.

[21] A. S. Karunajeewa, U. R. Abeyratne, and C. Hukins, "Multi-feature snore sound analysis in obstructive sleep apnea-hypopnea syndrome," Physiological Measurement, vol. 32, p. 83, 2011.

[22] U. R. Abeyratne, A. S. Karunajeewa, and H. Ghaemmaghami, "Multi-parametric analysis of snore sounds for the community screening of sleep apnea with nongaussianity index," United States Patent 20120004749, 2010.

[23] J. E. Markel and A. H. Gray, Linear prediction of speech. New York: Springer-Verlag Inc, 1982.

[24] R. G. Bachu, S. Kopparthi, B. Adapa, and B. D. Barkana, "Voiced/Unvoiced Decision for Speech Signals Based on Zero-Crossing Rate and Energy," in Advanced Techniques in Computing Sciences and Software Engineering, K. Elleithy, Ed., ed: Springer Netherlands, 2010, pp. 279-282.

[25] H. Ghaemmaghami, U. R. Abeyratne, and C. Hukins, "Normal probability testing of snore signals for diagnosis of obstructive sleep apnea," in Engineering in Medicine and Biology Society, 2009. EMBC 2009. Annual International Conference of the IEEE, 2009, pp. 5551-5554.

[26] El Safty S. and El Zonkoly A., "Applying wavelet entropy principle in fault classification," International Journal of Electrical Power \& Energy System, vol. 31, pp. 604-607, 2008.

[27] R. O. Duda, P. E. Hart, and D. G. Stork, Pattern classification, 2 ed.: WileyInterscience, 2000.

[28] A. Waibel, T. Hanazawa, G. Hinton, K. Shikano, and K. J. Lang, "Phoneme recognition using time-delay neural networks," Acoustics, Speech and Signal Processing, IEEE Transactions on, vol. 37, pp. 328-339, 1989.

[29] D. Nguyen and B. Widrow, "Improving the learning speed of 2-layer neural networks by choosing initial values of the adaptive weights," in Neural Networks, 1990., 1990 IJCNN International Joint Conference on, 1990, pp. 21-26 vol.3.

[30] M. Riedmiller and H. Braun, "A direct adaptive method for faster backpropagation learning: the RPROP algorithm," in Neural Networks, 1993., IEEE International Conference on, 1993, pp. 586-591 vol.1.

[31] A. Chang, "Pediatric Cough: Children Are Not Miniature Adults," Lung, vol. 188, pp. 33-40, 2010.

[32] S. Sung-Hwan, T. Hashimoto, and S. Hatano, "Automatic Detection System for Cough Sounds as a Symptom of Abnormal Health Condition," Information Technology in Biomedicine, IEEE Transactions on, vol. 13, pp. 486-493, 2009.

[33] K Kosasih, U Abeyratne, V Swarnkar, R Triasih. Wavelet Augmented Cough Analysis for Rapid Childhood Pneumonia Diagnosis. IEEE Transactions on Biomedical Engineering, vol 62 (2), pp. 1185 - 1194, 2014. 